\title{
Activating PIK3R2 Gene Mutation
}

National Cancer Institute

\section{Source}

National Cancer Institute. Activating PIK3R2 Gene Mutation. NCI Thesaurus. Code

C142118.

A change in the nucleotide sequence of the PIK3R2 gene that that results in constitutive activation of phosphatidylinositol 3-kinase regulatory subunit beta and its downstream signaling pathways. 